Jpn. J. Genet. (1987) 62, pp. 315-324

\title{
High order structure of Chironomus polytene chromosomes by scanning electron microscopy
}

\author{
By Toshihiko Taniguchi and Susumu Takayama \\ Biological Laboratory, School of Science, Kwansei Gakuin University, \\ Nishinomiya 662
}

(Received August 24, 1987)

\begin{abstract}
Polytene chromosomes isolated from Chironomus salivary gland cells were processed with the $\mathrm{OsO}_{4} / \mathrm{TCH}$ technique and examined by scanning electron microscopy (SEM) mainly using stereo techniques. When chromosomes were somewhat extended for preparation, band and interband regions became clearly visible. The bands appear to be dense masses of kinky fibers while the interband regions consist of the $30 \mathrm{~nm}$ chromatin fibers. On the other hand, in less extended chromosomes where band and interband regions were less distinctive, most of the component fibers appear to have coiled configurations and their diameters range 150 to more than $200 \mathrm{~nm}$. We conclude, therefore, that the natural chromonemata of the polytene chromosomes are about $200 \mathrm{~nm}$ in diameter rather $30 \mathrm{~nm}$ even in the interband regions. The polytene chromosomes seem to have a cable-like structure.
\end{abstract}

\section{INTRODUCTION}

Despite a large number of studies on cytogenetics as well as on the organization of the genome at the DNA level, our understanding of the chromatin fiber structure in eukaryotic chromosomes is still controversial. A number of models for the higher-order structure of the metaphase chromosomes have been presented (Dupraw 1965; Stubblefield and Wray 1971; Paulson and Laemmli 1977; Coming 1978; Okada and Comings 1979; Zatsepiona et al. 1983). In all of these models, however, the only agreement we have at present is the existence of $30 \mathrm{~nm}$ chromatin fibers, and the higher-order structure of the chromatin fibers in metaphase chromosomes still remains uncertain.

Recently Rattner and Lin (1985) have suggested that the metaphase packing is achieved by compaction through helical coiling of a $200-300 \mathrm{~nm}$ fiber which is further composed of looped $30 \mathrm{~nm}$ fibers. The present authors (Taniguchi and Takayama 1986) have also reported that metaphase chromosome preparations specially processed for scanning electron microscopy (SEM) show fairly homogeneous fibrous structures measuring about $200 \mathrm{~nm}$ in diameter which in turn appear to coiled $30 \mathrm{~nm}$ fibers.

Polytene chromosomes seem to provide useful information for the funda- 
mental understanding of the organization of chromatin fibers in metaphase chromosomes because of their structural characteristics, i. e., the occurrence of the reproducible patterns of bands and interbands, There have been number of studies on polytene chromosomes by transmission electron microscopy (TEM), but information on their whole structure available from these studies are rather limited. On the other hand, observations by SEM seem to make it possible to reveal the characteristic architecture of polytene chromosomes in a three dimensional figure. However, SEM studies on this type of chromosomes so far published are rather incomplete (Brady et al. 1977; Sedat and Manuelidis 1978; Iino and Naguro 1980; Mortin and Sedat 1982).

In this study, we applied our SEM technique (Taniguchi and Takayama 1986), which enabled us to show the presence of $200 \mathrm{~nm}$ fibrous structures in the mammalian metaphase chromosomes, to the Chironomus polytene chromosomes, and obtained some interesting findings on their chromatin fiber structure.

\section{MATERIALS AND METHODS}

Salivary gland of Chironomus plumosus larvae were isolated on glass slides and treated with $70 \%$ acetic acid for $15 \mathrm{~min}$. Under cover glasses, they were squashed carefully to isolate polytene chromosomes. After a brief wash in a $1 / 15 \mathrm{M}$ phosphate buffer solution ( $\mathrm{pH} \mathrm{6.8),} \mathrm{chromosomes} \mathrm{were} \mathrm{processed} \mathrm{for}$ SEM by the application of $\mathrm{OsO}_{4} /$ thiocarbohydrazide (TCH) technique (Ip and Fischman 1979; Harrison et al. 1982). Chromosomes were fixed with 3\% glutaraldehyde in the phosphate buffer solution for $30 \mathrm{~min}$, rinsed in the buffer three times, and further treated as follows: (i) fixation in $1 \% \mathrm{OsO}_{4}$ in the buffer for $30 \mathrm{~min}$ followed by three time rinses in distilled water; (ii) $20 \mathrm{~min}$ incubation in a freshly prepared and filtered saturated solution of TCH in distilled water followed by three time rinses in distilled water. These two steps were repeated more than three times. The specimens were then dehydrated through a graded ethanol series $(30 \%$ to $100 \%)$ and three changes of amyl acetate. Finally they were critical-point dried from liquid carbon dioxide with amyl acetate as the transitional fluid. After sputter coating with $10 \mathrm{~nm}$ of gold, chromosomes were viewed with the JEOL JSM-T200 SEM operated at $25 \mathrm{KV}$. Photographs were taken using stereo techniques.

\section{RESULTS}

The chromosome shown in pairs of stereo photographs in Figure 1 seems to have been somewhat extended as a result of preparative artifacts. There are two prominent cross bands and several much less conspicuous bands with interband regions between them. Such a topography is in a good agreement with the views so far reported by TEM. In interband regions, separated thin 


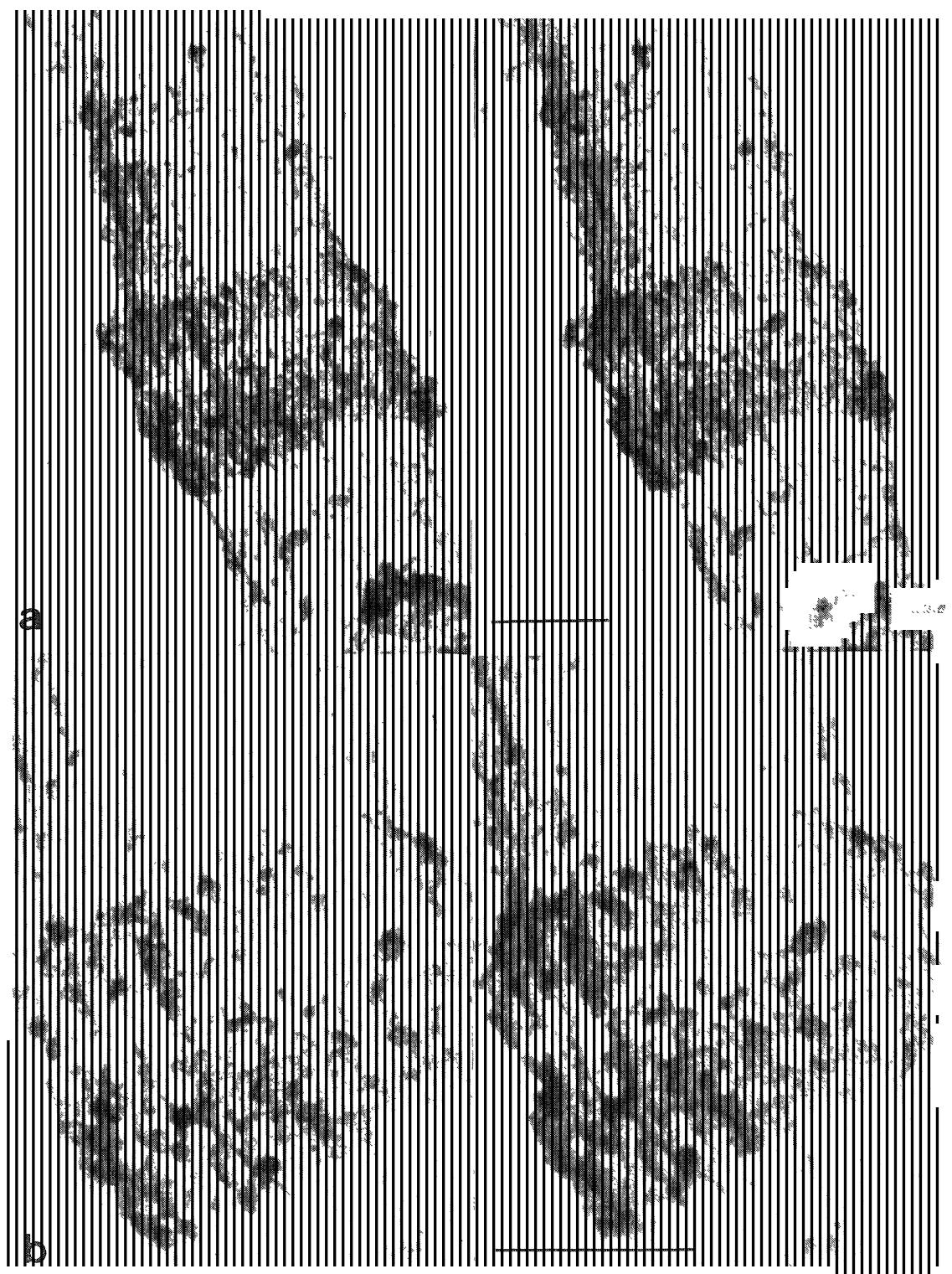

Fig. 1. Stereo pair scanning electron micrographs of part of a polytene chromosome from the Chironomus salivary gland cell. a: The chromosome appears somewhat extended, showing distinct band and interband regions. b: Part of the same chromosome as shown in a, showing highly prominent bands which consist of thicker fibers compared those in the adjacent interbands. Bars indicate $1 \mu \mathrm{m}$.

fibers which might correspond to $30 \mathrm{~nm}$ chromatin fibers are seen to run parallel to the long axis. Such fibers, however, frequently appear somewhat thicker than $30 \mathrm{~nm}$, since they tend to have some nodular or kinky appearances. 


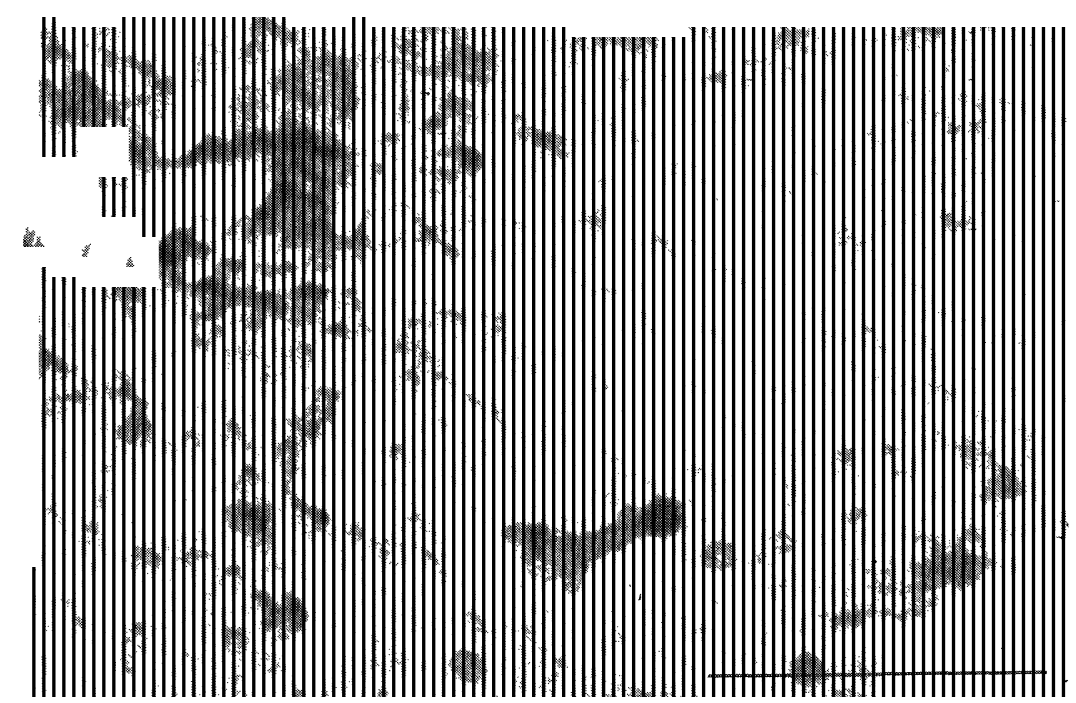

Ftg. 2. Part of a relatively extended chromosome; there are several minute bands and interbands gathering close to each other. Bar indicates $1 \mu \mathrm{m}$.

On the other hand, banded regions appear to consist of densely packed nodules or highly kinked fibers. The larger the bands are the more kinky they appear. Bands, therefore, can vary in a stereo viewer. This situation is clear in the two prominent band regions shown in Figure $1 \mathrm{~b}$. These band regions appear to consist of highly kinked and of raised fiber components which are fairly homogeneous in diameter measuring about $120 \mathrm{~nm}$. The difference in size is clearly revealed in the portion near the bottom of the Figure $1 \mathrm{~b}$, where the boundary between band and interband is visible.

Figure 2 is a portion of another chromosome. Considerable numbers of minute bands are distinguishable as small masses of kinky fibers which are located close to each other in a relatively small region.

The chromosome shown in Figure 3 seems somewhat less extended compared to the one in Figure 1 while the bundles of component fibers, chromonemata, appear twisted and disturbed to some extent. In this chromosome, most chromatin fibers appear clearly coiled even in the interband regions (Fig. $3 \mathrm{a}$ ). The diameters of such coiled fibers are roughly $130 \mathrm{~nm}$. Figure $3 \mathrm{~b}$ reveals a portion of another chromosome likewise less extended where all the chromatin fibers are seen to have distinctly coiled appearances.

Figure 4 shows a chromosome which seems little extended, probably revealing an appearance close to its native state. Although the topography appears to be reflecting the band-interband configuration, typical interband regions are no longer discernible in this chromosome. It appears to consist of nodular of kinky fibers which are considerably thicker than the fibers ob- 


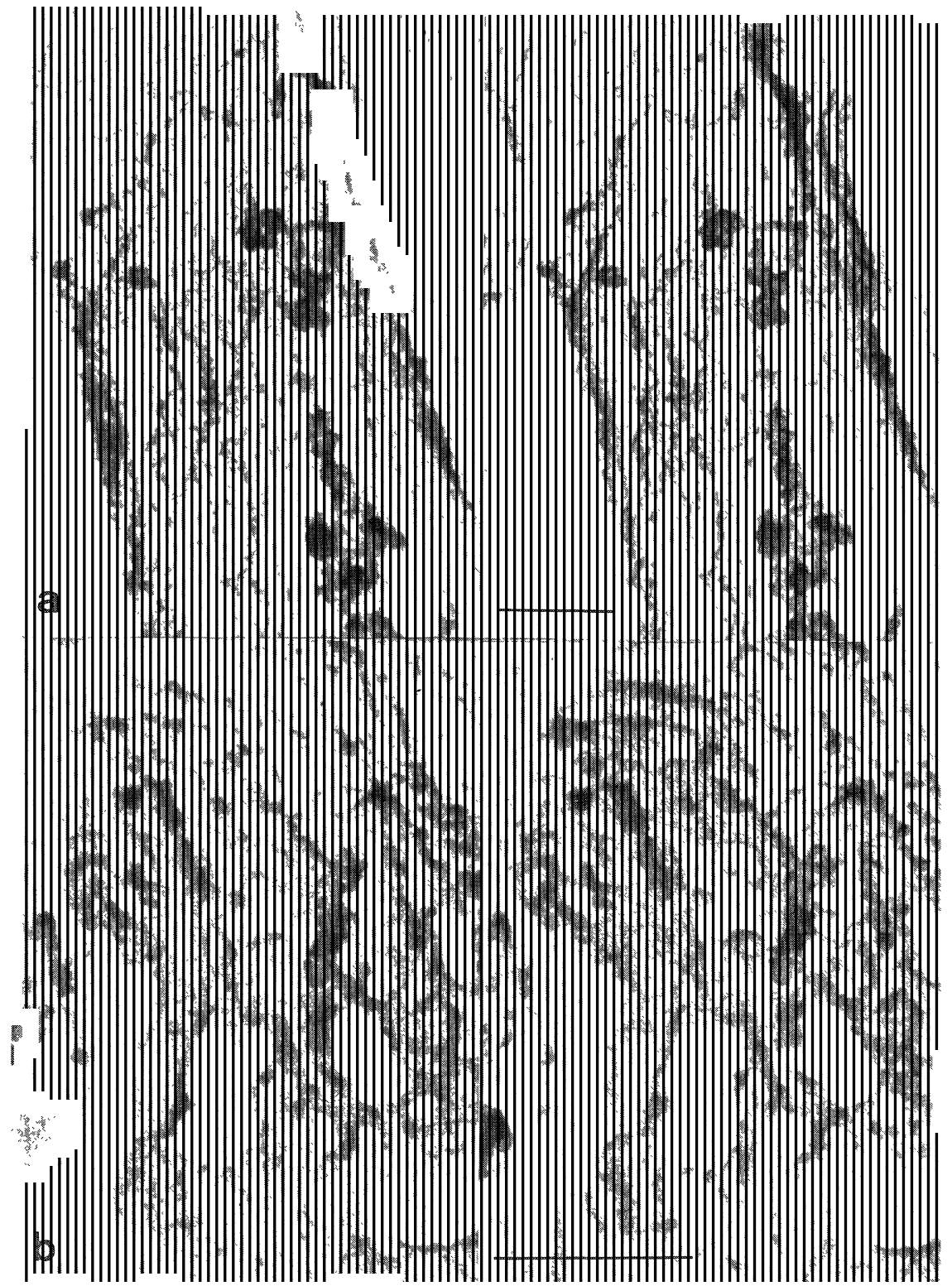

F'ig. 3. Stereo pair micrographs of part of a relatively less extended chromosomes. a: Most chromonemata appear clearly coiled. b: The similar situation is seen in another chromosome. Bars indicate $1 \mu \mathrm{m}$.

served in Figures 1-3 ranging from 150 to more than $200 \mathrm{~nm}$ in diameter (Fig. 4 b).

Figure 5 shows a chromosome with a broken end which probably happened to occur during the preparation. The appearance of this broken end seems to 


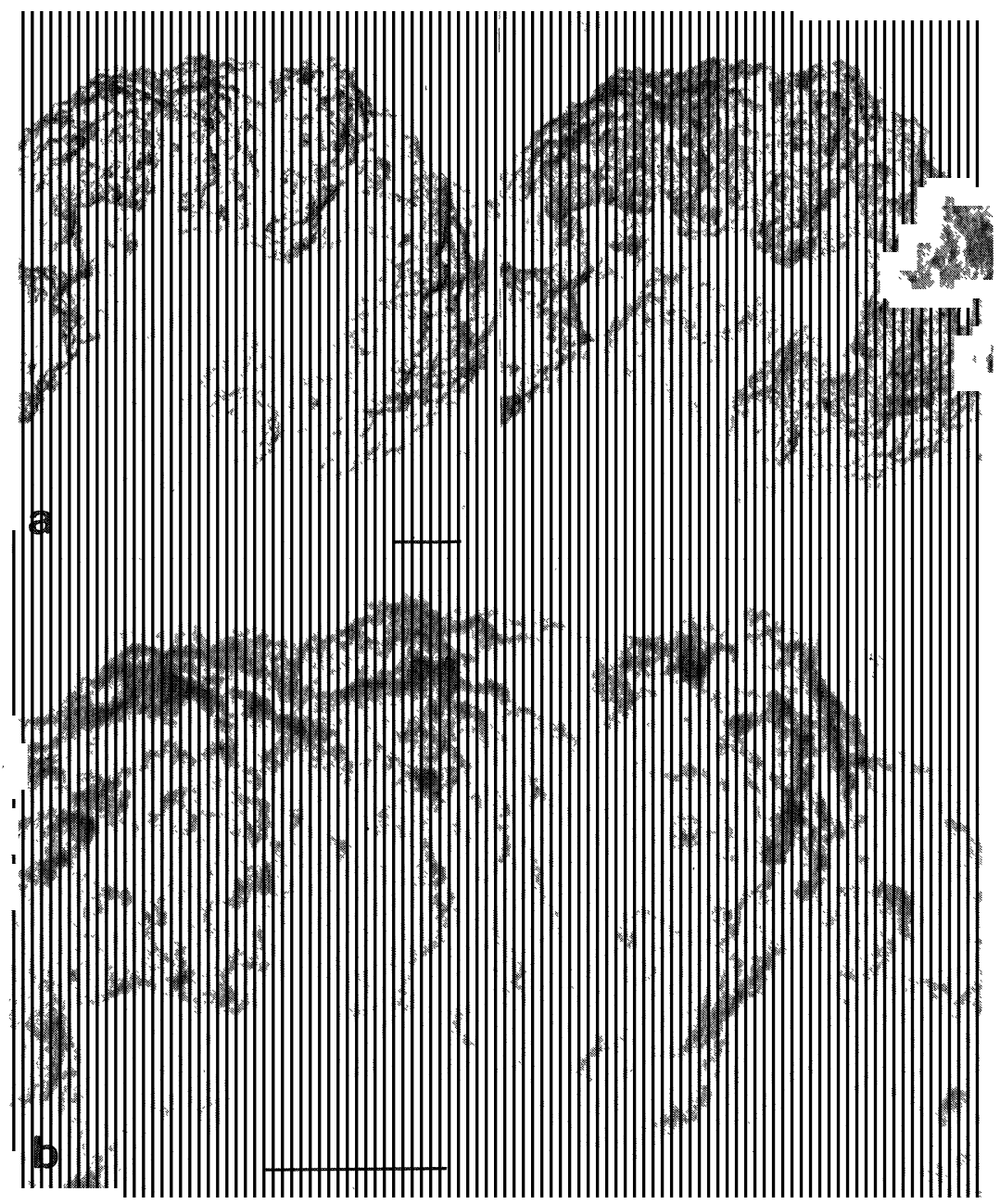

Fig. 4. Part of a chromosome little extended. a: Stereo pair micrographs. The bandinterband configuration appears obscure. b: Part of the same chromosome as shown in a. The thickness of the chromonemata ranges from 150 to more than $200 \mathrm{~nm}$ in diameter. Bars indicate $1 \mu \mathrm{m}$.

suggest that the polytene chromosome is made of fairly packed chromatin fibers without any hollow. Observations on some other chromosomes encountered in this study were also suggestive of the presence of such a cablelike architecture in them.

\section{DISCUSSION}

Our SEM method with stereo techniques have allowed to reveal the chromonema arrangement in both band and interband regions. The demonstration 


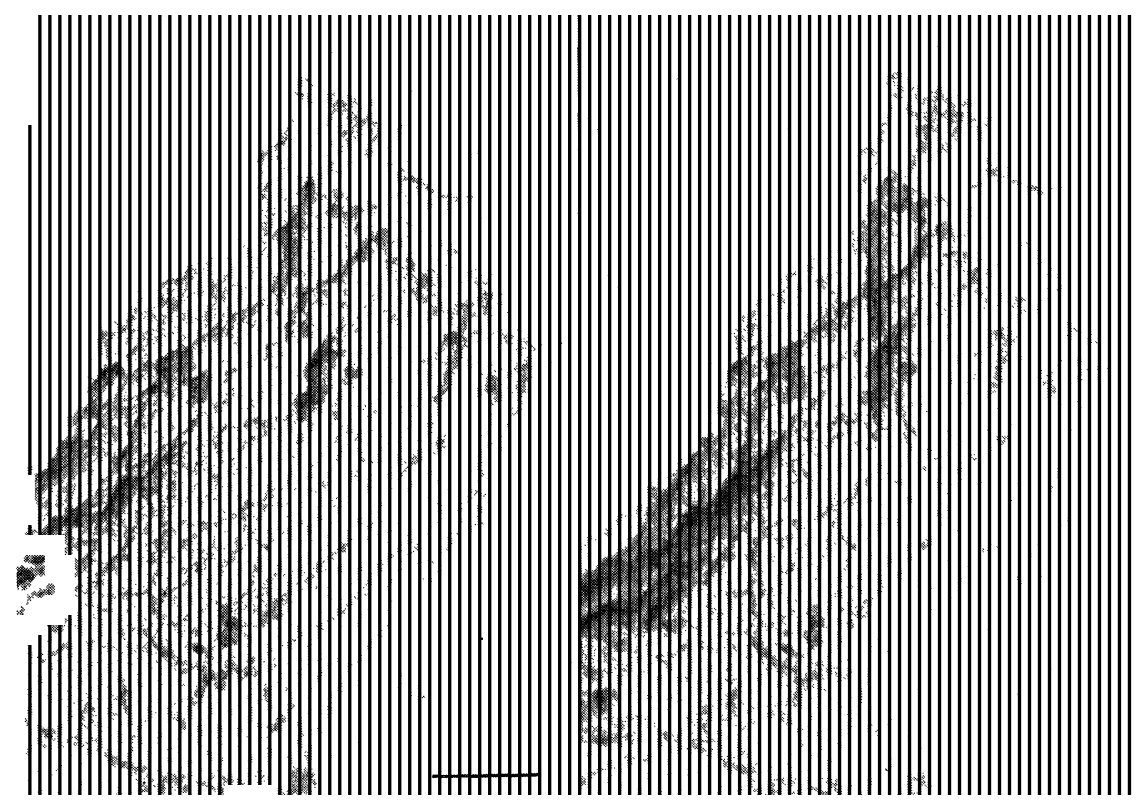

Fig. 5. Stereo pair micrographs of the broken end of a chromosome, being suggestive of the cable-like architecture. Bar indicates $1 \mu \mathrm{m}$.

of $200 \mathrm{~nm}$ fibers in the polytene chromosomes seems quite important since thick chromatin fibers of this size have been reported in the mammalian metaphase chromosomes by the present authors (Taniguchi and Takayama 1986). This has led us to assume that such $200 \mathrm{~nm}$ fibers may be a common high-order structure of chromatin fibers not only in the mammalian metaphase chromosomes but also in polytene chromosomes which are in general considered to be prophasic but not metaphasic. Since the coiled fibers shown in the present study were observed mainly in less or little extended chromosomes but not in extended ones, such a configuration might have been preserved only in the chromosomes prepared in the intention of keeping chromosomes as less extended as possible. TEM images of polytene chromosomes so far published are based on the highly extended chromosomes unless using sectioned materials. In such preparations, chromonemata in the interband reigns appear to be $30 \mathrm{~nm}$ chromatin fibers (Ananiev and Barsky 1985). In the present study, as reported in other SEM studies (Brady et al. 1977; Iino et al. 1980) we also found fibers roughly corresponding to the $30 \mathrm{~nm}$ fibers in the interband regions of extended chromosomes. On the other hand, the prominent band regions of less extended chromosomes appeared to consist of coiled $200 \mathrm{~nm}$ fibers.

On the basis of these findings, we tried to make a model for the polytene chromosome organization (Figure 6). In this model, the polytene chromosome 


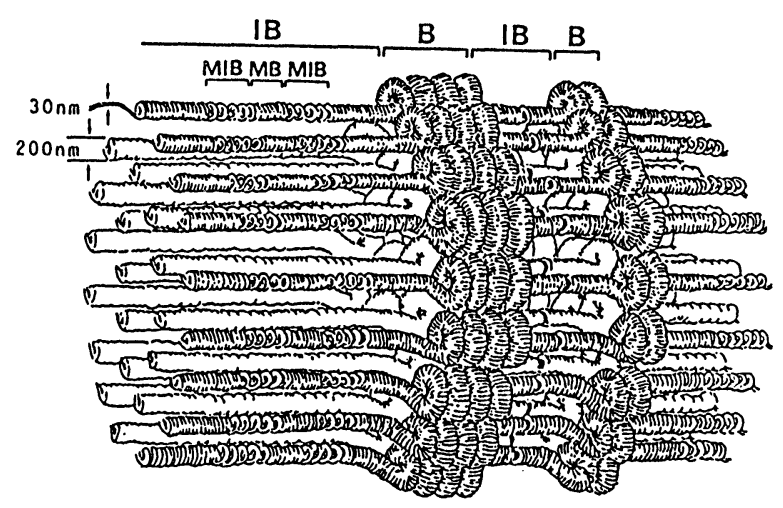

Fig. 6. Schematic representation of coiled coil model of the polytene chromosome. The $30 \mathrm{~nm}$ chromatin fibers coil to form $200 \mathrm{~nm}$ fibers and bands (B) occur as the result of further coiling of the $200 \mathrm{~nm}$ fibers while interbands (IB) contain minute bands (MB) and minute interbands (MIB) which result from local loosening of the $200 \mathrm{~nm}$ fibers.

is shown to be a cable-like bundle of chromonemata. The chromonemata basically assume $200 \mathrm{~nm}$ fibers which in turn result from coiling of $30 \mathrm{~nm}$ ones. Even the interband regions, therefore, consist of such coiled fibers whereas the coiling of $30 \mathrm{~nm}$ fibers may be loosened to various degrees in some portions. In other words, there is a possibility that a so-called interband between two bands (prominent bands) can contain minute bands and minute interbands as well. The clustered minute bands with the interbands seen in Figure 2 may represent some of the possible configurations resulting from such loosening.

The presence of band and interband configurations in polytene chromosomes is one of the most conspicuous characteristics under light microscopy. It is naturally impossible, however, to distinguish the presence of extremely minute bands and interbands in the conventionally stained preparations by light microscopy. In addition, it is true that the polytene chromosomes prepared for light microscopy are highly extended. Such artificial extension of the chromosomes seems to occur more severely in the whole-mounted preparations for TEM and thus minute bands would disappear in such preparations. It is, therefore, impossible to exactly correlate band and interband configurations in the stained preparations with those from TEM images. The necessity of TEM for the detection of "real" interbands in the sectioned materials has been claimed by Vlassova et al. (1985).

On the other hand, we interpret the prominent bands as the clusters of gyres resulting from coiling of $200 \mathrm{~nm}$ fibers. If this is true, the configuration of the prominent bands would correspond to that of the metaphase chromatid as the highest order of coiling in the hierarchies of chromatin condensation, 
and thus the chromonema organization of the polytene chromosome would be comparable to that of the mammalian metaphase chromosome (Taniguchi and Takayama 1986). However, if such gyres as the constituents of the prominent bands are too fragile to endure the preparative forces, they would become somewhat loosened and unwound. This may account for the fact that in the present study the prominent bands showed highly raised but complicated appearances in the fiber arrangement.

There have been considerable numbers of studies on the functional significance of interbands and some results are contradicting to each others. Some recent studies have claimed the transcriptional activity of interbands (Jamrich et al. 1977, Semenshin et al. 1979, 1986, Vlassova et al. 1985). This seems quite probable since decondensation of chromatin fibers should be at least related to transcriptional activity. However, it seems possible that some bands and interbands are convertible from one to the other and the minute bands and interbands mentioned above might belong to this type of structures. In this context, it is also possible that at least some, if not all, prominent bands are essentially different from minute bands in the chromatin constituents as well as in the function. In our model, these two types of bands are illustrated to be different at the level of the chromatin condensation.

\section{REFERENCES}

ANANiev, E. V. and BARSKy, V. E. (1985) Elementary structure in polytene chromosomes of Drosophila melanogaster. Chromosoma 93, 104-112.

Brady, T., Bailey, J. E. and PAYne, M. B. (1977) Scanning electron microscopy of isolated Chironomus polytene chromosomes. Chromosoma 60, 179-186.

Comings, D. E. (1978) Mechanisms of chromosome banding and implication for chromosome structure. Annu Rev Genet 12, 25-46.

Dupraw, E. J. (1965) Macromolecular organization of nuclei and chromosomes: a folded fibre model based on wholemount electron microscopy. Nature 206, 338-343.

Harrison, C. J., Allen, T. D., Britch, M. and Harris, R. (1982) High-resolution scanning microscopy of human metaphase chromosomes. J Cell Sci 56, 400-422.

Ino, A. and NAGURo, T. (1980) Polytene chromosomes observed by scanning electron microscope. Cytobios 27, 157-165.

IP, W. and Fischman, D. A. (1979) High resolution scanning electron microscopy of isolated in situ cytoskeletal elements. J Cell Biol 83, 249-253.

Jamrich, M., Greenleaf, A. L., Bautz, F. A. and Bautz, E. K. (1977) Functional oganization of polytene chromosomes. Cold Spring Harbor Quant Biol 42, 389-396.

Mortin, L. I. and Sedat, J. W. (1982) Structure of Drosophila polytene chromosomes. Evidence for a toroidal organization of the bands. J Cell Sci 57, 73-113.

OKada, T. A. and Comings, D. E. (1979) Higher order structure of chromosomes. Chromosoma $72,1-14$.

Paulson, J. R. and Laemmli, U. K. (1977) The structure of histone-depleted chromosomes. Cell $12,817-828$.

RAttNeR, J. B. and Lin, C. C. (1985) Radial loops and helical coils coexist in metaphase chromosomes. Cell 42, 291-296.

Sedat, J. W. and Manuelidls, L. (1978) A direct approach to the structure of eukaryotic chro- 
mosomes. Cold Spring Harbor Symp Biol 42, 331-350.

Semenshin, V. F., Zhimulev, I. F. and Belyaeva, E. S. (1979) Electron microscope autoradiographic study on transcriptional activity of Drosophila melanogaster polytene chromosomes. Chromosoma 73, 163-177.

Semenshin, V. F., Belyaeva, E. S., Shimulev, I. F., Lis, J. T., Ridhards, G. and Bourouis, M. (1986) Electron microscopical analysis of Drosophila polytene chromosomes. IV. Mapping of morphological structures appearing as a result of morphological structures appearing as a result of transformation of DNA sequences into chromosomes. Chromosoma 93, 461-368.

Stubblefield, E. and Wray, W. (1971) Architecture of the chinese hamster metaphase chromosome.Chromosoma 32, 262-294.

TANiguchi, T. and TAKAYAma, S. (1986) High-order structure of metaphase chromosomes: Evidence for a multiple coiling model. Chromosoma 93, 511-514.

Vlassova, I. E., Umbetova, G. H., Zimmermann, V. H., Alonso, C., Belyaeva, E. S. and ZhimUlev, I. F. (1985) Immunofluorescence localization of DNA: RNA hybrids in Drosophila melanogaster chromosomes. Chromosoma 91, 251-258.

Zatsepiona, O. V., Polyakov, V. Yu. and Chentsov, Yu. S. (1983) Chromonema and chromomere. Structural units of mitotic and interphase chromosomes. Chromosoma 88. 91-97. 